Article

\title{
Function Algebras on $r$-Discrete Abelian Groupoids
}

\author{
Hassan Myrnouri ${ }^{1, *}$ and Massoud Amini ${ }^{2}$ \\ 1 Department of Mathematics, Faculty of Mathematical Sciences, Lahijan Branch of Islamic Azad University, \\ Guilan, Iran \\ 2 Department of Mathematics, Faculty of Mathematical Sciences, Tarbiat Modares University, Tehran \\ 14115-134, Iran; mamini@modares.ac.ir \\ * Correspondence: myrnouri@iau-lahijan.ac.ir
}

\begin{abstract}
We study certain function algebras and their operator algebra completions on r-discrete abelian groupoids, the corresponding conditional expectations, maximal abelian subalgebras (masa) and eigen-functionals.
\end{abstract}

Keywords: abelian groupoid; function algebra; decomposable groupoid; eigen-functional

MSC: 22A22

\section{Introduction}

For a locally compact Hausdorff topological group $G$, the function algebra $C_{c}(G)$ consisting of all continuous functions of compact support on $G$ encodes all the information on the topology of $G$. For instance $G$ is compact iff $C_{c}(G)$ is unital. On the other hand it gives no information on the algebraic structure of $G$, since its algebraic operations are defined pointwise. Indeed, for such groups $G_{1}$ and $G_{2}$, the corresponding function algebras $C_{c}\left(G_{1}\right)$ and $C_{c}\left(G_{2}\right)$ are isomorphic (as normed algebras) iff $G_{1}$ is homeomorphic to $G_{2}$ as a topological space. To overcome this shortcoming, people usually endow $C_{c}(G)$ with the convolution product

$$
f * g(x)=\int_{G} f(y) g\left(y^{-1} x\right) d y,
$$

where the integral is taken against a left Haar measure (an essentially unique, left translation invariant, regular Borel measure) on $G$. With this product, $C_{c}(G)$ is again a normed algebra, but this time in the above situation, the function algebras $C_{c}\left(G_{1}\right)$ and $C_{c}\left(G_{2}\right)$ are isomorphic iff $G_{1}$ is homeomorphic and algebraically isomorphic to $G_{2}$ as a topological group. It now remains to complete the convolution algebra $C_{c}(G)$ to a Banach or complete operator algebra. One completion of the first kind is to the Banach algebra $L^{1}(G)$ of absolutely integrable functions on $G$ (with respect to the left Haar measure). This is a descent algebra whose representation theory fully reflects that of $G$, namely, its non-degenerate representations are in one-one correspondence with unitary representations of G [6]. The only major shortcoming of this function algebra is its spectral theory. Indeed, among Banach $*$-algebras, only $C^{*}$-algebras have a fairly good spectral theory. The good news is that one could complete $L^{1}(G)$ into a universal (full) $C^{*}$-algebra $C^{*}(G)$ with the same representation theory. This is done using all non-degenerate representations on Hilbert spaces and taking supremum of the corresponding operator norms. Alternatively, one could take the completion under the operator norm in the image of the left regular representation to get the reduced $C^{*}$-algebra $C_{r}^{*}(G)$. The two constructions are the same exactly when $G$ is amenable [15]. The above mentioned full and reduced $C^{*}$-algebra construction is generalized by Jean Renault [18] to locally compact (not necessarily Hausdorff) groupoids. The construction is shown to be very useful in the study of the representation theory of groupoids [1]. 
In this paper we study $C^{*}$-algebras on abelian groupoids and the corresponding extensions. We also study eigen-functionals on abelian groupoids which paly the role of characters in group $C^{*}$-algebras. The structure of abelian groupoids is recently studied by the authors in $[13,14]$. Our basic reference for groupoids is [18].

A groupoid $\Gamma$ is a small category in which each morphism is invertible (for a formal definition, see [18]). The unit space $X=\Gamma^{(0)}$ of $\Gamma$ is the subset of elements $\gamma \gamma^{-1}$ where $\gamma$ ranges over $\Gamma$. The range source maps $s, r: \Gamma \rightarrow \Gamma^{(0)}$ are defined by $r(\gamma)=\gamma \gamma^{-1}, s(\gamma)=\gamma^{-1} \gamma$, for $\gamma \in \Gamma$. For $u \in \Gamma^{(0)}$, we set $\Gamma^{u}=r^{-1}(u)$ and $\Gamma_{u}=s^{-1}(u)$. The isotropy group of $\Gamma$ at a unit $u$ is $\Gamma_{u}^{u}=\{\gamma \in \Gamma: r(\gamma)=s(\gamma)\}$. For subgroupoids $\mathcal{H}$ and $\mathcal{K}$ of $\Gamma, \Gamma / \mathcal{K}$ (resp. $\mathcal{H} \backslash \Gamma$ ) is the set of right (resp. left) classes in $\Gamma$ modulo $\mathcal{K}$ (resp. $\mathcal{H}$ ), that is,

$$
\left\{\gamma \mathcal{K}^{s(\gamma)} \mid \gamma \in \Gamma\right\}\left\{\mathcal{H}_{r(\gamma)} \gamma \mid \gamma \in \Gamma\right\}
$$

The quotient spaces $\Gamma / \mathcal{K}$ and $\mathcal{H} \backslash \Gamma$ are fibered on $\Gamma^{(0)}$. Note that even for the case of a group bundle $\mathcal{H}$, it is not necessary that each fibre $\mathcal{H}(x)$ is open. Indeed we have the following characterization for the case of wide subgroupoids (i.e., a subgroupoid whose unit space is the same as the unit space of the groupoid).

Proposition 1. Every wide subgroupoid $\mathcal{H}$ with open fibers $\mathcal{H}^{x}$ in a groupoid $\Gamma$ with locally compact topology is closed.

Proof. Since $\Gamma-\mathcal{H}$ is equal to the disjoint union of the cosets of $\mathcal{H}$ in $\Gamma$, except $\mathcal{H}$ itself, and these cosets are open (because the fibers $\mathcal{H}^{x}$ are open and the multiplication is continuous), $\Gamma-\mathcal{H}$ is open and so $\mathcal{H}$ is closed.

A subgroupoid $\mathcal{N}$ of $\Gamma$ is called normal if there exist a groupoid $\mathcal{G}$ and a surjective groupoid morphism $\pi: \Gamma \rightarrow \mathcal{G}$ such that $\pi^{-1}\left(\mathcal{G}^{(0)}\right)=\mathcal{N}$. In this case, $\Gamma / \mathcal{N}$ and $\mathcal{G}$ are isomorphic, as groupoids. As a typical example, $\Gamma(X)$ is a closed normal subgroupoid of locally compact groupoid $\Gamma$. It is also a locally compact group bundle [18, page 18]. When $\Gamma$ is locally compact, the subgroupoid $\Gamma(X)$ is closed, but not necessarily open. Note that $\operatorname{in} t \Gamma(X)$ is an open subgroupoid, but it has open bundle maps only if $\Gamma$ has a continuous Haar system, in which case, int $\Gamma(X)$ also has a continuous Haar system.

For the case of group bundles, a normal locally compact (resp. measured) subgroupoid $\mathcal{N}$ of a locally compact (resp. measured) groupoid $\Gamma$ is a continuous (resp. measurable) field $\{x \in X \rightarrow$ $\mathcal{N}(x) \subset \Gamma(x)\}$ of subgroups such that $\gamma \mathcal{N}(x) \gamma^{-1}=\mathcal{N}(y)$, where $\gamma: x \rightarrow y$ [8, Def 1.10].

It is shown in [2, Prop. 1.1.10] that the quotient topology on $\Gamma / \mathcal{N}$ is induced by the continuous surjection $\pi: \Gamma \rightarrow \Gamma / \mathcal{N}$. For a topological groupoid $\Gamma$ with subgroupoid $\mathcal{N}$ (with the induced topology) if $\Gamma$ is second countable and locally compact and $\mathcal{N}$ is locally closed, then $\mathcal{N}$ is locally compact. If $\mathcal{N}$ is open in $\Gamma$, then its range and source maps are open.

The subgroupoids $\Gamma(X)$ and $R(\Gamma)$ are measured groupoids. Note that for a measured groupoid, the continuity of the Haar systems $\beta$ and $\alpha$ holds only in particular cases (for example see [11] and [7]). In these cases, the above groupoids are locally compact groupoids.

\section{Main results}

Definition 2. An abelian groupoid is a groupoid whose isotropy groups are abelian.

If $\Gamma$ is $r$-discrete and $X$ is compact, $C_{r}^{*}(\Gamma)$ is unital, and its unit is the characteristic function $X$ [16, page 15].

We consider the trivial continuous $\Gamma$ - bundle of $C^{*}$-algebras over $X$ with fibre $\mathbb{C}$ and apply [1, Prop. 6.1.10]. If $\Gamma$ is an abelian groupoid with amenable associated principal groupoid $R(\Gamma)$, then $C^{*}(\Gamma)=C_{r e d}^{*}(\Gamma)$. 
Lemma 3. If $\Gamma$ is an $r$-discrete abelian groupoid with finite unit space, then $C^{*}(\Gamma)=C_{r e d}^{*}(\Gamma)$.

Proof. Because an $r$-discrete abelian groupoid with finite unit space is decomposable abelian groupoid [13], and therefore amenable, and for amenable groupoid $\Gamma$, we have $C^{*}(\Gamma)=C_{\text {red }}^{*}(\Gamma)$ [1, Prop. 6.1.10].

Theorem 4. If $\Gamma$ is an $r$-discrete abelian groupoid with finite unit space, then $\left(C^{*}(\Gamma), C^{*}(\Gamma(X))\right)$ is a $C^{*}$-diagonal pair.

Proof. Since $\left(\widehat{\Gamma(X)} \rtimes_{c} R\right)^{(0)}=\widehat{\Gamma(X)}$ and $\widehat{\Gamma(X)} \rtimes_{c} R$ is a principal r-discrete groupoid,

$$
\left(C^{*}\left(\widehat{\Gamma(X)} \rtimes_{C} R, \mathcal{G}\right), C_{0}(\widehat{\Gamma(X)})\right)
$$

is a diagonal pair [10, Theorem VIII.6], hence $\left(C^{*}(\Gamma), C^{*}(\Gamma(X))\right)$ is a $C^{*}$-diagonal, because the isomorphism between groupoid $C^{*}$-algebras preserves the diagonal.

Corollary 5. If $\Gamma$ is an $r$-discrete abelian groupoid with finite unit space, then the restriction map $P: C^{*}(\Gamma) \rightarrow$ $C^{*}(\Gamma(X))$ is the unique faithful conditional expectation onto $C^{*}(\Gamma(X))$.

In [11], the authors show that there is generalized conditional expectation $C^{*}(\Gamma) \rightarrow C^{*}(\Gamma(x))$, if we add these generalized conditional expectations, we get a generalized conditional expectation $C^{*}(\Gamma) \rightarrow C^{*}(\Gamma(X))$. In [4, Theorem 3.4], the following corollary is proved for the $r$-discrete case.

Corollary 6. If $\Gamma$ is an $r$-discrete abelian groupoid with finite unit space, then $C^{*}(\Gamma(X))\left(r e s p . C_{\mu}^{*}(\Gamma(X))\right)$ is a masa in $C^{*}(\Gamma)\left(\operatorname{resp} . C_{\mu}^{*}(\Gamma)\right)$.

If $\Gamma$ is a nontrivial $r$-discrete abelian groupoid with finite unit space, $X$ can not be the interior of $\Gamma(X)$, therefore $C^{*}(X)$ is not a maximal subalgebra of $C^{*}(\Gamma)$ [18, Prop. II.4.7(ii)]. But $C^{*}(\Gamma(X))$ is commutative, and we can conclude that there is no $f \in C_{c}(\Gamma)$ in the center of the commutative $C^{*}$-algebra $C^{*}(\Gamma(X))$ with support outside of $\Gamma(X)$. The next result follows from [10, Remark VIII5].

Corollary 7. If $\Gamma$ is an $r$-discrete abelian groupoid with finite unit space, $C^{*}(\Gamma(X))$ has the extension property in $C^{*}(\Gamma)$

Corollary 8. If $\Gamma$ is an $r$-discrete abelian groupoid with finite unit space, $C^{*}(\Gamma)$ is regular as a $C^{*}(\Gamma(X))$-bimodule.

For a Banach $C^{*}(\Gamma(X))$-bimodule $\mathcal{M}$, An element $m \in \mathcal{M}$ is called an intertwiner if $m . C^{*}(\Gamma(X))=C^{*}(\Gamma(X))$. $m$. If $m \in \mathcal{M}$ is an intertwiner such that for every $f \in C^{*}(\Gamma(X)), f . m \in$ $\mathbb{C} m$, we call $m$ a minimal intertwiner. When the abelian algebra is a masa, intertwiners are the same as normalizers [5, Prop 3.3], that is the set $N\left(C^{*}(\Gamma(X))\right)=\left\{v \in C^{*}(\Gamma): v C^{*}(\Gamma(X)) v^{*} \subset\right.$ $C^{*}(\Gamma(X))$ and $\left.v^{*} C^{*}(\Gamma(X)) v \subset C^{*}(\Gamma(X))\right\}$. For $v \in N\left(C^{*}(\Gamma(X))\right)$, put $\operatorname{dom}(v):=\left\{\phi \in \mathcal{Z}: \phi\left(v^{*} v\right)>\right.$ $0\}$, this is an open set in $\mathcal{Z}=C^{*}(\overline{\Gamma(X)})$. As observed by Kumjian, there is a homeomorphism $\beta_{v}: \operatorname{dom}(v) \rightarrow \operatorname{dom}\left(v^{*}\right):=\operatorname{ran}(v)$ given by

$$
\beta_{v}(\phi)(f)=\frac{\phi\left(v^{*} * f * v\right)}{\phi\left(v^{*} * v\right)},
$$

such that $\beta_{v}^{-1}=\beta_{v^{*}}$.

Regularity of a bimodule $\mathcal{M}$ is equivalent to norm-density of the set of $C^{*}(\Gamma(X))$-intertwiners. Intertwiners and normalizers are closely related, at least when $C^{*}(\Gamma(X))$ is a masa in the unital $C^{*}$-algebra $C^{*}(\Gamma)$ containing $\mathcal{M}[5$, Remark 4.2]: 
(i) If $v \in C^{*}(\Gamma)$ is an intertwiner for $C^{*}(\Gamma(X))$, then $v^{*} v, v v^{*} \in C^{*}(\Gamma(X))^{\prime} \cap C^{*}(\Gamma)$. If $C^{*}(\Gamma(X))$ is maximal abelian in $C^{*}(\Gamma)$, then $v$ is a normalizer of $C^{*}(\Gamma(X))$ [5, proposition 3.3].

(ii) For $v \in N\left(C^{*}(\Gamma(X))\right)$, if $\beta_{v^{*}}$ extends to a homeomorphism of $\operatorname{dom}\left(v^{*}\right)$ onto $\operatorname{dom}(v)$, then $v$ is an intertwiner. Moreover, if $I$ is the set of intertwiners, then $N\left(C^{*}(\Gamma(X))\right)$ is contained in the norm-closure of $I$, and when $C^{*}(\Gamma(X))$ is a masa in $C^{*}(\Gamma), N\left(C^{*}(\Gamma(X))\right)=I[5$, Proposition 3.4].

Definition 9. A minimal intertwiner of $\mathcal{M}^{*}$ will be called an eigen-functional, when necessary for clarity, we use $C^{*}(\Gamma(X))$-eigen-functional. That is, a $C^{*}(\Gamma(X))$-eigen-functional is a nonzero linear functional $\phi: \mathcal{M} \rightarrow \mathbb{C}$ such that, for all $f \in C^{*}(\Gamma(X)), g \rightarrow \phi(f * g), g \rightarrow \phi(g * f)$ are multiples of $\phi$. We equip the set of all $C^{*}(\Gamma(X))$-eigen-functionals $\mathcal{E}_{C^{*}(\Gamma(X))}(\mathcal{M})$ in the relative weak* topology $\sigma\left(\mathcal{M}^{*}, \mathcal{M}\right)$.

We know that $\widehat{C^{*}(\Gamma(X)} \subset \mathcal{E}^{1}(\mathcal{M})$. If $\Gamma=G$ is an abelian group, we have $C^{*}(\Gamma(X))=C^{*}(\Gamma)=$ $C^{*}(G)$, hence $\mathcal{E}_{C^{*}(\Gamma(X))}^{1}\left(C^{*}(\Gamma)\right)=\widehat{C^{*}(G)}$ hence eigen-functionals are generalizations of multiplicative functionals.

Given an eigen-functional $\phi \in \mathcal{E}_{C^{*}(\Gamma(X))}(\mathcal{M})$, the associativity of the maps $f \in C^{*}(\Gamma(X)) \mapsto f . \phi$ and $f \in C^{*}(\Gamma(X)) \mapsto \phi . f$ yields the existence of unique multiplicative linear functionals $s(\phi)$ and $r(\phi)$ on $C^{*}(\Gamma(X))$ satisfying $s(\phi)(f) \phi=f . \phi$ and $r(\phi)(f) \phi=\phi . f$, that is,

$$
\phi(g * f)=\phi(g)[s(\phi)(f)], \phi(f * g)=[r(\phi)(f)] \phi(g) .
$$

We call $s(\phi)$ and $r(\phi)$ the source and range of $\phi$, respectively [5, page 6].

There is a natural action of the nonzero complex numbers $z$ on $\mathcal{E}(\mathcal{M})$, sending $(z, \phi)$ to the functional $m \mapsto z \phi(m)$; clearly $s(z \phi)=s(\phi)$ and $r(z \phi)=r(\phi)$. Also, $\mathcal{E}(\mathcal{M}) \cup\{0\}$ is closed in the weak*-topology. Furthermore, $r: \mathcal{E}(\mathcal{M}) \rightarrow C^{*}(\widehat{\Gamma(X)})$ and $s: \mathcal{E}(\mathcal{M}) \rightarrow C^{*}(\Gamma(X))$ are continuous.

Notation 10. We put $\mathcal{G}=\mathcal{E}^{1}\left(C^{*}(\Gamma)\right)$, where $\mathcal{E}^{1}\left(C^{*}(\Gamma)\right)$ is the collection of norm-one eigenvectors for the dual action of $C^{*}(\Gamma(X))$ on the Banach space dual $C^{*}(\Gamma)^{*}$, also for a bimodule $\mathcal{M} \subset C^{*}(\Gamma), \mathcal{G} \mid \mathcal{M}$ is defined directly in terms of the bimodule structure of $\mathcal{M}$, without explicit reference to $C^{*}(\Gamma)$ as in [5, Remark 4.16].

The groupoid $\mathcal{G}$, with suitable operations and the relative weak*- topology, admits a natural $\mathbb{T}$-action. If $\phi, \psi \in \mathcal{E}(\mathcal{M})$ satisfy $r(\phi)=r(\psi)$ and $s(\phi)=s(\psi)$, then there exists $z \in \mathbb{C}$ such that $z \neq 0$ and $\phi=z \psi[5$, Corollary 4.10].

With the relative weak*-topology, $\mathcal{E}^{1}(\mathcal{M}) \cup\{0\}$ is compact [5, Prop. 4.17]. Thus, $\mathcal{E}^{1}(\mathcal{M})$ is a locally compact Hausdorff space. As usual, we may regard an element $m \in \mathcal{M}$ as a function on $\mathcal{E}^{1}(\mathcal{M})$ via $\hat{m}(\phi)=\phi(m)$. When $\mathcal{A}$ is both a norm-closed algebra and a $C^{*}(\Gamma(X))$-bimodule, the coordinate system $\mathcal{E}^{1}(\mathcal{A})$ has the additional structure of a continuous partially defined product as described in [5, Remark 4.14]. In this case, we will sometimes refer to the coordinate system as a semitwist.

Definition 11. Let $\mathcal{R}(\mathcal{M}):=\left\{|\phi|: \phi \in \mathcal{E}^{1}(\mathcal{M})\right\}$. Then $\mathcal{R}(\mathcal{M})$ may be identified with the quotient $\mathcal{E}^{1}(\mathcal{M}) \backslash \mathbb{T}$ of $\mathcal{E}^{1}(\mathcal{M})$ by the natural action of $\mathbb{T}$. A twist is a proper $\mathbb{T}$-groupoid $\mathcal{G}$ so that $\mathcal{G} \backslash \mathbb{T}$ is a principal r-discrete groupoid. The topology on $\mathcal{R}\left(C^{*}(\Gamma)\right)$ is compatible with the groupoid operations, so $\mathcal{R}\left(C^{*}(\Gamma)\right)$ is a topological equivalence relation.

Consider the $C^{*}$-diagonal $\left(C^{*}(\Gamma), C^{*}(\Gamma(X))\right)$, and let $\mathcal{M} \subset C^{*}(\Gamma)$ be a norm closed $C^{*}(\Gamma(X))$-bimodule. Then the span of $\mathcal{E}^{1}(\mathcal{M})$ is $\sigma\left(\mathcal{M}^{*}, \mathcal{M}\right)$-dense in $\mathcal{M}^{*}$. Suppose $\mathcal{A}$ is a norm closed algebra satisfying $C^{*}(\Gamma(X)) \subset \mathcal{A} \subset C^{*}(\Gamma)$. If $\mathcal{B}$ is the $C^{*}$-subalgebra of $C^{*}(\Gamma)$ generated by $\mathcal{A}$, then $\mathcal{B}$ is the $C^{*}$-envelope of $\mathcal{A}$. If in addition, $\mathcal{B}=C^{*}(\Gamma)$, then $\mathcal{R}\left(C^{*}(\Gamma)\right)$ is the topological equivalence relation generated by $\mathcal{R}(\mathcal{A})$ [5]. 
Eigen-functionals can be viewed as normal linear functionals on $\mathcal{C}^{* *}$ and we start by using the polar decomposition for such functionals one obtains a minimal partial isometry for each eigen-functional as follows. By the polar decomposition for linear functionals, there is a partial isometry $u^{*} \in C^{*}(\Gamma)^{* *}$ and positive linear functionals $|\phi|,\left|\phi^{*}\right| \in C^{*}(\Gamma)^{*}$ so that $\phi=u^{*} \cdot|\phi|=\left|\phi^{*}\right| \cdot u^{*}$. We find that $r(\phi)=|\phi|$ and $s(\phi)=\left|\phi^{*}\right|$. Moreover, $u u^{*}$ and $u^{*} u$ are the smallest projections in $C^{*}(\Gamma)^{* *}$ which satisfy, $u^{*} u \cdot s(\phi)=s(\phi) \cdot u^{*} u=s(\phi)$ and $u u^{*} \cdot r(\phi)=r(\phi) \cdot u u^{*}=r(\phi)$. For $\phi \in \mathcal{E}^{1}\left(C^{*}(\Gamma)\right)$, we call the above partial isometry $u$, the partial isometry associated to $\phi$ and denote it by $v_{\phi}$. If $\phi \in C^{*}(\widehat{\Gamma(X)})$, then $u$ is a projection and is denoted by $p_{\phi}$. The above equations show that $v_{\phi}^{*} v_{\phi}=p_{s(\phi)}$ and $v_{\phi} v_{\phi}^{*}=p_{r(\phi)}$. Moreover, given $\phi \in \mathcal{E}^{1}\left(C^{*}(\Gamma)\right), v_{\phi}$, may be characterized as the unique minimal partial isometry $w \in C^{*}(\Gamma)^{* *}$ such that $\phi(w)>0$. Recall that $\chi, \xi \in C^{*}(\hat{\Gamma}(X))$ satisfy $(\chi, \xi) \in \mathcal{R}\left(C^{*}(\Gamma)\right)$ if and only if there is $\phi \in \mathcal{E}^{1}\left(C^{*}(\Gamma)\right)$ with $r(\phi)=\chi$ and $s(\phi)=\xi$. For brevity, we write $\chi \sim \xi$ in this case [5].

For $\chi \in C^{*}(\widehat{\Gamma(X)})$, we use $\left(H_{\chi}, \pi_{\chi}\right)$ for the GNS representation of $C^{*}(\Gamma)$ associated to the unique extension of $\chi$. Let $\chi, \xi \in C^{*}(\Gamma(X))$, then $\xi \sim \chi$ if and only if the GNS representations $\pi_{\chi}$ and $\pi_{\xi}$ are unitarily equivalent [5, Lemma 5.8]. Therefore if $\chi, \xi \in C^{*}(\widehat{\Gamma(X)})(x)$, then $\xi \sim \chi$ iff $\xi=\chi$. If we set $\mathcal{M}=\left\{f \in C^{*}(\Gamma): \chi\left(f^{*} f\right)=0\right\}$, then $C^{*}(\Gamma) / \mathcal{M}$ is complete relative to the norm induced by the inner product $\langle f+\mathcal{M}, g+\mathcal{M}\rangle=\chi\left(g^{*} f\right)$, and thus $H_{\chi}=C^{*}(\Gamma) / \mathcal{M}[11$, Lemma 2.11].

The next result is from [5].

Proposition 12. Suppose $\chi \in C^{*}(\widehat{\Gamma(X)})$ and $\phi \in \mathcal{E}^{1}\left(C^{*}(\Gamma)\right)$ satisfy $\chi \sim s(\phi)$. Then there exist unique orthogonal unit vectors $\omega_{1}, \omega_{2} \in H_{\chi}$ such that for every $f \in C^{*}(\Gamma), \phi(f)=\left\langle\pi_{\chi}(f) \omega_{1}, \omega_{2}\right\rangle$.

Theorem 13. We have $\left.\mathcal{R}\left(C^{*}(\Gamma)\right) \cong C^{*} \widehat{(\Gamma(X)}\right) \rtimes_{C} R(\Gamma)=\mathcal{Z} \rtimes_{c} R$, algebraically and topologically.

Proof. Ionescu and Williams showed that every representation of $\Gamma$ induced from an irreducible representation of a stability group is irreducible [7]. We can extend a character $\chi \in C^{*}(\Gamma(x))$ to $\chi \in \widehat{C^{*}(\Gamma)}$ such that $\chi \mid C^{*}\left(\left.\Gamma\right|_{[y] \neq[x]}\right)=0$, and since the extension is unique in $C^{*}$-diagonals, this extension will be equal to $\operatorname{Ind}\left(x, \Gamma(X)_{x}, \chi\right)$. Let $\chi, \xi \in \widehat{\Gamma(X)}$, then by $[3$, lem 2.11$] \chi \sim \xi$ if and only if the GNS representations $\pi_{\chi}$ and $\pi_{\xi}$ are unitarily equivalent. By [11, lemma 2.5], $\operatorname{Ind}\left(x, \Gamma(X)_{x}, \chi\right)$ is unitarily equivalent to $\operatorname{Ind}\left(x . k, \Gamma(X)_{s(k)}, \chi . k\right)$ in $\widehat{C^{*}(\Gamma)}$ so they are in the same class in $\widehat{C^{*}(\Gamma)}$, that is $\chi \sim \chi$. $k$ where $k \in R$. But if two stability groups of $\Gamma$ are not in the same orbit, none of their irreducible representation can be equivalent. Therefore the two space are the same. Finally, since the topology is r-discrete, they are equal topologically.

Corollary 14. The groupoid $\mathcal{G}$ is the $\mathbb{T}$-groupoid of $\mathcal{Z} \rtimes_{c} R$, that is we have the exact sequence

$$
\mathcal{Z} \rightarrow \mathcal{Z} \times \mathbb{T} \rightarrow \mathcal{G} \rightarrow \mathcal{G} \backslash \mathbb{T} \cong \mathcal{Z} \rtimes_{c} R
$$

An $r$-discrete abelian groupoid with finite unit space and open fibers $\Gamma(x)$ has $\operatorname{AF}$ principal groupoid [13].

Lemma 15. If $\Gamma$ is an $r$-discrete abelian groupoid whose principal groupoid $R$ is $A F$, then $C^{*}(\Gamma)$ is $A F$.

For $A=C^{*}(\Gamma)$ in the above lemma, we can find an $\mathrm{AF}$ groupoid $\mathcal{R}$ such that $C^{*}(\Gamma) \cong C^{*}(\mathcal{R})$. We conjecture that $\mathcal{R}=\widehat{\Gamma(X)} \rtimes_{c} R$. This is true by [13, Proposition 2.3], when $\Gamma$ has a finite unite space.

Definition 16. Let $P$ be an open subset of $\mathcal{R}$ containing $\widehat{\Gamma(X)}$. Then $P$ is called a partial order in $\mathcal{R}$ if $P \circ P \subset P$ and $P \cap P^{-1}=\widehat{\Gamma(X)}$. If moreover, $P \cup P^{-1}=\mathcal{R}$ then $P$ is called a total order in $\mathcal{R}$. If 
$P \circ P \subset P$ and $P=P^{-1}$, then $P$ is an equivalence relation on a subgroupoid of $\mathcal{R}$. If $P$ is a total order on $\mathcal{R}$ then $P$ is closed.

If $\mathcal{A}$ is a strongly maximal triangular subalgebra of a unital $\mathrm{AF} C^{*}$-algebra $C^{*}(\Gamma)$, then $C^{*}(\Gamma(X))=\mathcal{A} \cap \mathcal{A}^{*}$ is a canonical masa in $C^{*}(\Gamma)$ and $\mathcal{A}+\mathcal{A}^{*}$ is dense in $C^{*}(\Gamma)$.

We now coordinatize the triple of algebras $\left(C^{*}(\Gamma(X)), \mathcal{A}, C^{*}(\Gamma)\right)$, where $C^{*}(\Gamma)$ is an $\mathrm{AF} C^{*}$ algebra and $\mathcal{A}$ is a strongly maximal triangular subalgebra of $C^{*}(\Gamma)$ whose diagonal is $C^{*}(\Gamma(X))$. We define the spectral triple $(\mathcal{Z}, P, \mathcal{R})$ for $\left(C^{*}(\Gamma(X)), \mathcal{A}, C^{*}(\Gamma)\right)$.

Theorem 17. For each partial order $P$ in $\mathcal{R}, \mathcal{A}(P)=\left\{f \in C^{*}(\Gamma) \mid\right.$ supp $\left.f \subseteq P\right\}$ is a norm closed subalgebra of $C^{*}(\Gamma)$ containing $C^{*}(\Gamma(X))$. Conversely, each subalgebra $A$ of $C^{*}(\Gamma)$ containing $C^{*}(\Gamma(X))$ is of the form $\mathcal{A}(P)$, for a unique partial order. The correspondence $P \mapsto \mathcal{A}(P)$ is an inclusion preserving bijection between the collection of partial orders in $\mathcal{R}$ and norm closed subalgebras of $C^{*}(\Gamma)$ containing $C^{*}(\Gamma(X))$.

\section{Conclusion}

In this paper, we studied $C^{*}$-algebras of $r$-discrete abelian groupoids. When the unit space is finite, we showed that the full and reduced $C^{*}$-algebras are the same. In this case, there is a conditional expectation onto the $C^{*}$-algebra of the principal groupoid. We also studied maximal abelian subalgebras (masa) and eigen-functionals for such groupoids.

\section{Authors contributions}

$\mathrm{HM}$ and MA have participated and prepared the manuscript. Both authors read and approved the final manuscript.

\section{Author details}

1. Assistant Professor, Department of Mathematics, Faculty of Mathematical Sciences, Lahijan Branch of Islamic Azad University, Guilan, Iran.

2. Professor, Department of Mathematics, Faculty of Mathematical Sciences, Tarbiat Modares University, Tehran 14115-134, Iran

\section{Acknowledgements}

The authors were supported by a grant from Islamic Azad University, Lahijan. The authors would like to thank the referee for useful comments.

\section{Competing interests}

The authors declare that they have no competing interests.

\section{References}

1. Anantharaman-Delaroche, C.; Renault, J. Amenable Groupoids; Monographie de L'enseignement Mathematique 36: Geneve, 2000.

2. Cadet, F. Déformation et quantification par groupoïde des variétés toriques, Docteure de L'universite D'orleans, thèse; 2001.

3. Daenzer, C. A groupoid approach to noncommutative T-duality, Ph.D thesis, University of Pennsylvania; 2007.

4. Deaconu, V.; Kumjian, A.; Ramazan, B. Fell bundles associated to groupoid morphisms. Math. Scand. 2008 102 (2), 305-319.

5. Donsig, A. P.; Pitts, D. R. Coordinate systems and bounded isomorphisms. J. Operator Theory 2008, 59, 359-416.

6. Folland, G.B. A course in abstract harmonic analysis. CRC Press: Boca Raton, 1994.

7. Goehle, G. Groupoid crossed products, Ph.D dissertation, Dartmouth College, 2009. 
8. Katayama, Y. and Takesaki,M. Outer actions of a discrete amenable group on approximately finite dimensional factors II, The III $_{\lambda}$-case, $\lambda \neq 0$. Math. Scand. 2007 100, 75-129.

9. Muhly, P. S. Coordinates in Operator Algebras, unpublished manuscript.

10. Muhly, P. S. private communication.

11. Muhly, P. S.; Renault, J.; Williams, D. P. Continuous-trace groupoid $C^{*}$-algebras III. Trans. Amer. Math. Soc. 1996348 (9), 3621-3641.

12. Muhly, P. S.; Solel, B. Subalgebras of groupoid C*-algebras. J. Reine Angew. Math. 402, 41-75.

13. Myrnouri, H.; Amini, M. Decomposable abelian groupoids. International Mathematical Forum 201048 , 2371-2380.

14. Myrnouri, H.; Amini, M. Matched pairs in abelian groupoids. Gulf Journal of Mathematics 2014 2, $22-29$.

15. Pedersen, G.K. C ${ }^{*}$-algebras and their automorphism groups, Academic Press: New York, 1979.

16. Phillips, N.C. Crossed products of the cantor set by free minimal actions of $\mathbb{Z}^{d}$. Comm. Math. Phys. 2005 256, 1-42.

17. Renault, J. Cartan subalgebras in $C^{*}$-algebras. Irish Math. Soc. Bull. 2008 61, 29-63.

18. Renault, J. A groupoid approach to $C^{*}$-algebras, Lecture notes in Mathematics 793, Springer Verlag: Berlin, 1980.

19. Suzuki, H. Central $S^{1}$-extensions of symplectic groupoids and the poisson classes. Pacific J. Math. 2002 203, 489-501.

20. Tu, J. L. Groupoid cohomology and extensions. Trans. Amer. Math. Soc. 2006 358, 4721-4747.

21. Vallin, J. M. Actions and coactions of finite quantum groupoids on von Neumann algebras, extensions of the match pair procedure. J. of Algebra 2007 314, 789-816.

22. Yamagami, S. On factor decomposition of an ergodic groupoid. Kyoto Univ. Math. J. 1988 24, 379-395.

(C) 2016 by the authors; licensee Preprints.org, MDPI, Basel, Switzerland. This article is an open access article distributed under the terms and conditions of the Creative Commons Attribution (CC-BY) license (http:/ / creativecommons.org/licenses/by/4.0/). 